\title{
Predicting G-Quadruplexes from DNA Sequences Using Multi-Kernel Convolutional Neural Networks
}

\author{
Mira Barshai \\ mirabar@post.bgu.ac.il \\ School of Electrical and Computer Engineering \\ Ben-Gurion University of the Negev \\ Beer-Sheva, Israel
}

\author{
Yaron Orenstein \\ yaronore@bgu.ac.il \\ School of Electrical and Computer Engineering \\ Ben-Gurion University of the Negev \\ Beer-Sheva, Israel
}

\begin{abstract}
G-quadruplexes are nucleic acid secondary structures that form within guanine-rich DNA or RNA sequences. G-quadruplex formation can affect chromatin architecture and gene regulation and has been associated with genomic instability, genetic diseases and cancer progression. G-quadruplex formation in a DNA template can be assessed using polymerase stop assays, which measure polymerase stalling at G-quadruplex sites. An experimental technique, called G4-seq, was developed by combining features of the polymerase stop assay with Illumina next-generation sequencing. The experimental data produced by this technique provides unprecedented details on where and at what intensity do G-quadruplexes form in the human genome. Still, running the experimental protocol on a whole genome is an expensive and time-consuming process. Thus, it is highly desirable to have a computational method to predict G-quadruplex formation of new DNA sequences or whole genomes. Here, we present a new method, called G4detector, to predict G-quadruplexes from DNA sequences based on multi-kernel convolutional neural networks. To test G4detector, we compiled novel high-throughput in vitro and in vivo benchmarks. On these data, we show that G4detector outperforms extant methods for the same task on all benchmark datasets. We visualize the most important features of G4detector models and discover that G-quadruplex formation is highly depended on G-tracts length, their spacing and nucleotide composition between them. The code and benchmarks are publicly available on github.com/OrensteinLab/G4detector.
\end{abstract}

\section{CCS CONCEPTS}

- Computing methodologies $\rightarrow$ Supervised learning by classification; Neural networks; • Applied computing $\rightarrow$ Bioinformatics;

\section{KEYWORDS}

G-quadruplex, Convolutional neural networks

\section{ACM Reference format:}

Mira Barshai and Yaron Orenstein. 2019. Predicting G-Quadruplexes from DNA Sequences Using Multi-Kernel Convolutional Neural Networks. In

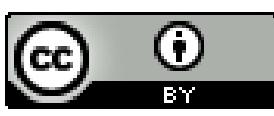

This work is licensed under a Creative Commons Attribution International 4.0 License.

ACM-BCB '19, September 7-10, 2019, Niagara Falls, NY, USA

(C) 2019 Copyright held by the owner/author(s).

ACM ISBN 978-1-4503-6666-3/19/09.

https://doi.org/10.1145/3307339.3342133
Proceedings of 10th ACM International Conference on Bioinformatics, Computational Biology and Health Informatics, Niagara Falls, NY, USA, September 7-10, 2019 (ACM-BCB '19), 10 pages.

https://doi.org/10.1145/3307339.3342133

\section{INTRODUCTION}

The formation of DNA secondary structures can influence biological processes such as replication, translation and splicing $[5,10]$. Single stranded DNA undergoes Hoogsteen base pairing between stretches of guanine nucleotides (G-tracts) to form four-stranded structures known as a G-quadruplexes (G4s) $[27,29,32]$ that occur in vivo [25]. G4 secondary structures arise in guanine-rich sequences where four guanine bases interact to form planar Gtetrads, which can self-stack [11] (Figure 1). Nucleotide sequences containing four tracts of three or more guanines separated by loops of variable length can also form G4s spontaneously in vitro and are stabilized by potassium $[23,27,36]$. Several non-canonical G4s have been described including those with longer loops, interrupted or mismatches in G-tracts (bulges) [34], indicating that sequence patterns are less stringent then previously thought. G4 formation is kinetically fast, and these structures are thermodynamically stable under physiological conditions, particularly in the presence of $\mathrm{K}^{+}[11]$.

Recent studies using immunofluorescence to visualize G4s revealed their active formation in human cells and tissues and highlighted potential functional implications of these structures in diseases such as cancer $[3,4,16]$. G4-forming DNA sequences have been identified in promoter regions of oncogenes $[13,15]$ and are conserved across species [21]. G4 formation has been implicated in genetic regulation of oncogenes such as c-MYC [30], and are a potential therapeutic target for clinical intervention $[7,8]$.

Computational predictions using simple algorithms suggest that over 300,000 sequence motifs of the type

$$
[G]^{3+}[A C G T]^{1-7}[G]^{3+}[A C G T]^{1-7}[G]^{3+}[A C G T]^{1-7}[G]^{3+}
$$

in the human genome have the potential to form a G4 structure [18, 33]. A more recent algorithm predicted that the number of potential G4 sequences is substantially higher [2]. These computational studies predict that G4 motifs are enriched in telomeres, promoters and in the first intron of genes, but they highlight the need to generate explicit experimental data about the existence and function (or functions) of G4s in biologically relevant contexts.

The prevalence and distribution of G4s in the human genome is a key question that is currently addressed primarily on the basis of simple computational prediction, such as regular expressions [12, 18, 19]. However, several experimental techniques were developed 


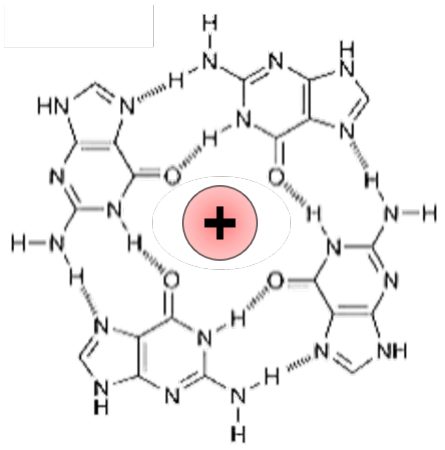

(a)

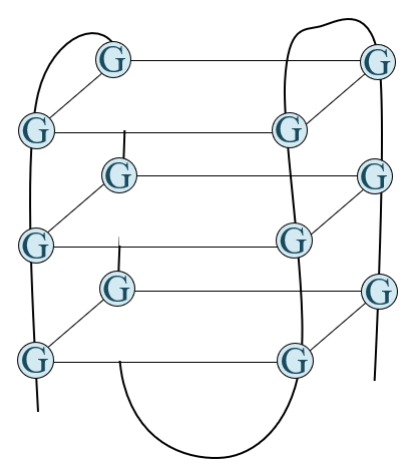

(b)

Figure 1: G-quadruplex (G4) DNA structure.

(a) A structural representation of a G-tetrad that makes up the core of G4 structures. The structure is stabilized by a cation. (b) A schematic representation of a single strand DNA sequence forming a G4.

to measure G4 formation in high-throughput. G4 formation in a DNA template can be assessed using polymerase stop assays, which measure polymerase stalling at G4 sites [35]. A method, called G4-seq, was developed by combining features of the polymerase stop assay with Illumina next-generation sequencing [9]. In this technology, DNA is sequenced from primary human B lymphocytes under conditions that either promote or disfavor G4 formation. Because polymerase stalling at G4 sites was found to affect base calling, sequencing readouts from both conditions can be compared to elucidate the exact position of G4 structures. Two independent approaches are used to promote DNA G4 stabilization: adding $K^{+}$or the G4-stabilizing ligand pyridostatin (PDS, $1 \mu \mathrm{M}$ ) to the sequencing buffers [9].

Although computational predictions coupled with G4-seq provide a framework for understanding the potential for G4 structure formation in genomes, it is important to map G4 DNA formation in situ. A step towards this goal is the use of G4 structure-specific antibodies as probes. G4-selective probes have been developed and employed to capture G4s by chromatin immunoprecipitation followed by sequencing (ChIP-seq). This technology, termed G4 ChIP-seq, was successfully applied to human cells to detect G4 formation in vivo in a genome-wide manner [14]. But still, both of these experimental protocols are time-consuming and expensive and require an experienced lab technician to run them. Thus, computational predictions are desired to replace the need for these experiments or at least profoundly reduce the list of G4 candidates for further testing using higher-resolution low-throughput assays.

Several methods were developed to tackle the challenge of predicting G4 formation in silico. One class of methods search for regular expression that contain four guanine stretches. The most recent one is G4hunter [2, 6, 22]. The main downside of these type of methods is that they do not rely on the high-throughput experimental data that was recently produced in multiple studies $[9,14]$. The other type of computational methods learn a predictive model from these experimental datasets. To the best of our knowledge, only one such method exists, Quadron, which aims to predict the strength (i.e., stability) of the G-quadruplex structure [28]. The label it predicts is the mismatch score which corresponds to the strength of the G4 structure. Still, no method was developed to classify DNA sequences for having the potential to form G4s or not.

In this study, we fill this gap by developing a new method, called G4detector, to classify G4 forming structures based on highthroughput experimental data. G4detector uses multi-kernel convolutional neural networks to predict $\mathrm{G} 4$ formation potential by sequence features. We compile novel benchmark datasets to test G4detector on both in vitro and in vivo data. On these benchmarks we compare G4detector to three different methods developed for the same task. Last, we visualize the important features of the model learned by G4detector and inspect their biological significance.

\section{MATERIALS AND METHODS}

\subsection{Datasets}

We generated novel datasets of high-throughput G-quadruplex measurements for benchmarking different computational methods for the prediction task. First, we used genomic coordinates as retrieved by G4-seq and G4 ChIP-seq experiments, performed in vitro [9] and in vivo [14] (GEO accession numbers GSE63874 and GSE76688, respectively). We extracted the corresponding DNA sequences using bedtools and based on the hg19 genome [26]. Since the in vitro sets included extremely short or long sequences, we removed the $10 \%$ of shortest and longest sequences. The results were three in vitro sets (corresponding to K, PDS and K_PDS stabilizers) and one in vivo. We reverse complemented the minus strand of the in vitro data and merged the set of sequences with the set of the plus strand. The in vivo data lacked strand information, so if a sequence contained more C's than G's, we used its reverse complement instead. We referred to those as the positive sets in the classification problem.

For each such positive set we created three different negatives sets. First, for each positive sequence we extracted a corresponding sequence of the same length from a random coordinate in the same chromosome. Second, for each positive sequence we shuffled its nucleotides by preserving dinucleotide frequencies [1]. Last, we looked for $\mathrm{G} 4$ subsequences by the following regular expression:

$$
[G]^{3+}[A C G T]^{1-7}[G]^{3+}[A C G T]^{1-7}[G]^{3+}[A C G T]^{1-7}[G]^{3+}
$$

and centered them around their native genomic context. The length of each such sequence was randomly picked to follow the length distribution of the positive sequence set. 


\subsection{G4detector neural network}

The deep learning model of G4detector is a multi-kernel convolutional neural network, previously proposed by Zhang el al. [37]. Since the G4 structures and the loops that comprise them vary in size, using a kernel of fixed size might not be beneficial for identifying the features that characterize a G4 structure. Instead, our method, G4detector, employs three parallel one-dimensional convolution layers of length 2, 3 and 6 and of depth (i.e., number of kernels) 80, 80 and 96, respectively. The output of each layer goes through a global max-pooling layer. These outputs are then concatenated to form a single numerical vector of length $80+80+96=256$. This vector is the input to a fully connected layer of 32 hidden nodes with a ReLU activation function. We did not test other parameters combinations, as G4detector already outperformed extant methods on this combination. Finally, the output is governed by a single neuron with a sigmoid activation function, which receives its input from the hidden layer. A sigmoid function assigns to each input DNA sequence a probability of forming a G4 structure. The architecture of G4detector is described in Figure 2.

G4detector was implemented using Keras library with Tensorflow backend. Training was preformed on GeForce 1080Ti GPU. The models were trained over 15 training epochs. Learning rate for all the models was set to 0.0001 and the batch size chosen was 128. The network's weights were randomly initialized using a normal distribution. We used Adam optimizer [20] with $\beta_{1}=0.9$ and $\beta_{2}=0.99$. For the model trained on the random, dishuffled and PQ datasets, average training times for each epoch were 70.31, 71.93 and 67.73 seconds, respectively.

\subsection{Comparison to previous methods}

We compared G4detector to three other methods. First, we compared to GraphProt [24] in the same manner as we used G4detector, i.e., the first test was 10 -fold cross validation on the in vitro data and the second test was training and testing on in vitro and in vivo sets, respectively. While GraphProt was originally developed to predict protein-RNA binding based on sequence and structure, on a sequence only mode it serves as very effective $k$-mer based approach to classify based on sequence features. For cross-validation, training and prediction, we ran GraphProt in the following manner: "per 1 GraphProt.pl -action <action> -onlyseq" with the appropriate sequence files. The actions were ' $c v$ ', 'train' and 'predict', respectively.

Second, we tested our method, G4detector, against trained models of two methods developed for the specific task of G-quadruplex

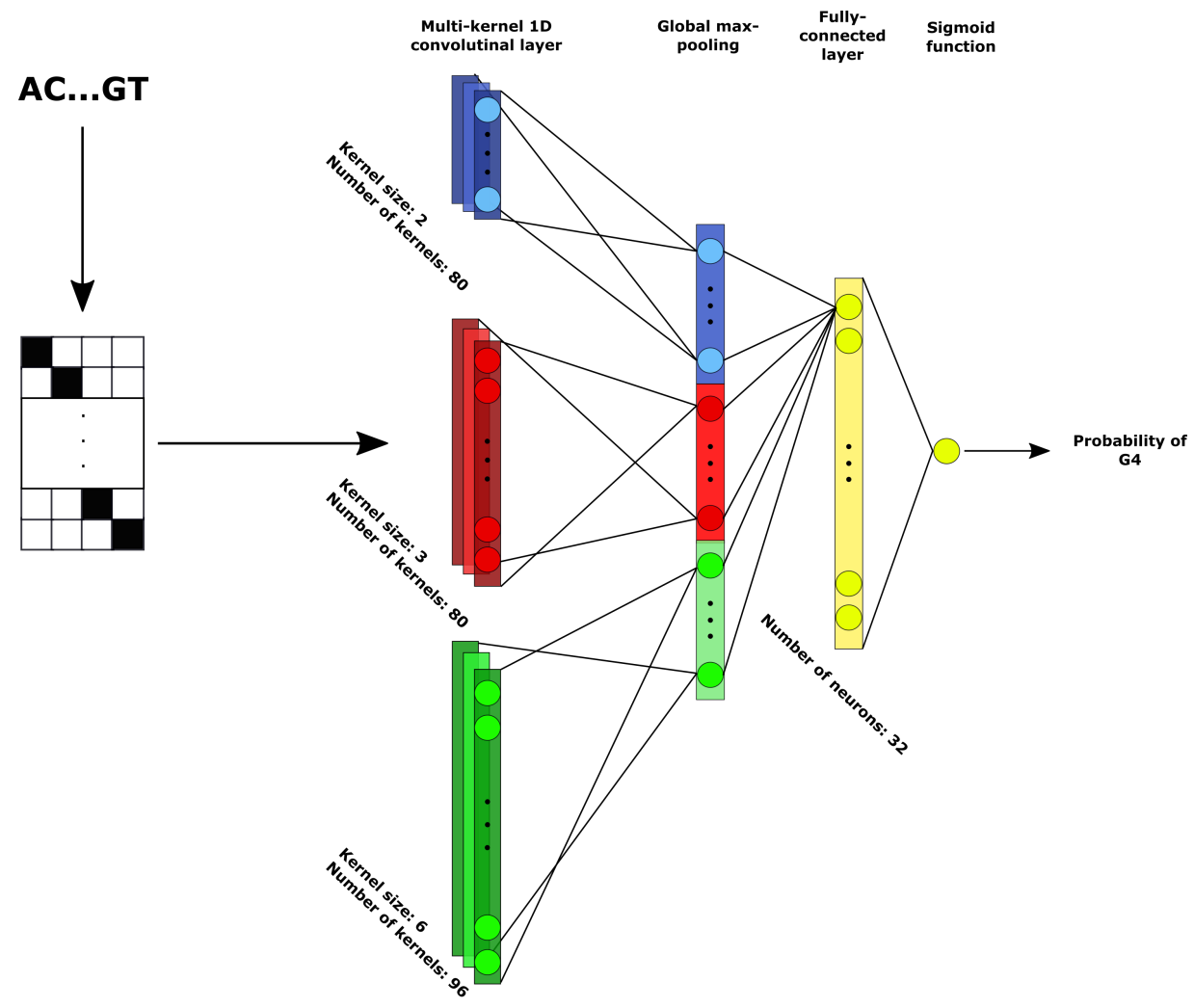

Figure 2: G4detector neural network to detect G-quadruplexes.

One-hot encoded DNA sequence is the input to G4detector. It is first processed in parallel by three convolutional layers of different sizes.

The outputs of each kernel go through a global-max pooling layer. The per-kernel maximums are then concatenated. The vector of concatenated maximums goes into a fully connected layer of 32 neurons, followed by a single output neuron. A score is assigned to each sequence, indicating the probability of it forming a G-quadruplex. 
identification: Quadron [28] and G4Hunter [2, 6, 22]. We ran Quadron with the default parameters. For each sequence that was not assigned a score by Quadron, we assigned it a zero score, as Quadron only predicts positive scores.

We implemented G4Hunter in python. In our implementation, we used a window of 25 bases, as it was set in [2], and a threshold of zero, to include all subsequences identified as G4s. The score given to the complete sequence is that of highest scored subsequence. If no G4 had been identified in a given sequence, its scores it the maximum over all its calculated windows. Since we already resolved the strand based on the experimental data, we were only looking for G4s on the forward strand. Thus, we ignored any G4 subsequence on the reverse strand. Only sequences of length at least the window size were given as input to our implementation of G4Hunter.

\subsection{Model visualization}

We used the integrated gradients approach as implemented in [17] to identify key features within a given sequence [31]. This approach attributes a deep learning network's prediction to features of the input relative to a neutral baseline and assigns a score to each such feature, that indicates its importance. The method computes the path integral of the gradients along a straight path between the input and the baseline. We used the all-zero matrix baseline.

To visualize the preferences learned by the G4detector model, we chose one positive sequence and visualized the attribution scores given to each feature (i.e., nucleotide) in the sequence. The gradient acts as a sensitivity map that tells us what the model is paying attention to. It should be noted here that the input to the model are one-hot vectors, which are a continuous relaxation of the original discrete sequences. Even though the model treats the input space as continuous, it is useful to think of the direction of the gradient at a given position being an indicator of a nucleotide switch at that position resulting in either an increase or decrease in the overall loss. The size of the letters in our visualizations corresponds to the relative importance of the given position of the input to the output, with positions more relevant to the final predictions having a greater size.

In addition, we visualized the interactions between $\mathrm{G} 4$ positions using a heat map. For this aim, we perform in silico mutagenesis. We use a specific input sequence as a reference and mutate it. First, we create all single nucleotide polymorphisms (SNPs). Then, we also generate all double nucleotide polymorphismps (DNPs). To identify interactions, we look for deviations from the addivitive model. Each element $(i, j)$ in the heatmap is the sum of differences of
SNP mutations minus the difference created by the DNP mutation. Each such element contains $4 \times 4$ cells representing all possible mutations from one nucleotide to the other. Formally, for positions $i, j$ and nucleotides $x, y$ the value of cell $(i, j, x, y)$ is:

$$
\triangle S N P(i, x)+\triangle S N P(j, y)-\triangle D N P(i, j, x, y)
$$

where $\triangle S N P(i, x)$ is the difference from the reference in predicted probability when mutating position $i$ to nucleotide $x$, and $\triangle D N P(i, j, x, y)$ is the difference from the reference in predicted probability when mutating positions $i, j$ to nucleotides $x, y$, respectively.

\section{RESULTS}

\subsection{Novel G4-quadruplex benchmark datasets}

We compiled novel benchmarks, based on the high-throughput in vitro and in vivo data generated by G4-seq [9] and G4 ChIP-seq [14] experiments, respectively, to train and test different computational methods for the task of G4 prediction. Three G4 stabilizers were used in G4-seq: K, PDS and K_PDS. This resulted in three different sets of genomic coordinates of G4-forming DNA sequences.

We turned each of the three sets into a classification problem by augmenting it with a negative set. Since each negative set may have its drawbacks, we used three different kinds of negatives:

(1) random: random genomic sequences

(2) dishuffle: randomly shuffled positives while preserving dinucleotide frequencies

(3) $P Q$ : predicted G-quadruplexes in the human genome according to a regular expression

The rationale behind the different negatives is as follows. First, random genomic sequences mimic the ability of our method to identify G4 sequences in a genome-wide manner, i.e. distinguishing them from 'regular' genomic sequences. Second, the shuffled sequences test a more difficult scenario, where the method needs to distinguish between sequences with similar local nucleotide statistics, but different orderings that make up G4s. Third, the PQ negative set is used to test the ability of the method to identify G4 DNA sequences that do not comply with the prior assumption of a G4 regular expression. We observed only $18.8 \%$ overlap with the positive set, which means that predictions by the regular expression are far from experimental measurements.

The in vivo G4 ChIP-seq positive set was augmented by three different negatives, similar to the in vitro case. For sequence and set statistics, see Table 1. For complete details of sequence set generation, see Subsection 2.1.

Table 1: Statistics of positive and negative sets of the novel benchmarks generated in this study.

\begin{tabular}{l|l|c|c|l}
\hline Class & Experiment & Number of sequences & Sequence lengths [nt] & Nucleotide frequency [\%] \\
\hline \hline Positive & G4-seq in vitro K & 398,966 & $256 \pm 38$ & A:25.5 C:21.4 G:30.4 T:22.8 \\
Positive & G4-seq in vitro PDS & 534,696 & $212 \pm 50$ & A:24.8 C:21.0 G:34.0 T:20.1 \\
Positive & G4-seq in vitro K_PDS & 327,170 & $188 \pm 44$ & A:24.3 C:20.4 G:35.0 T:20.3 \\
\hline Positive & G4 ChIP-seq in vivo & 8,954 & $266 \pm 132$ & A:16.0 C:30.1 G:40.2 T:14.7 \\
\hline Random negative & all experiments & as positive set & as positive set & A:29.8 C:20.2 G:20.2 T:29.8 \\
\hline Dishuffeled negative & all experiments & as positive set & as positive set & as positive set \\
\hline PQ negative & in silico & 375,782 & $221 \pm 52$ & A:24.5 C:21.2 G:34.9 T:19.4 \\
\hline
\end{tabular}




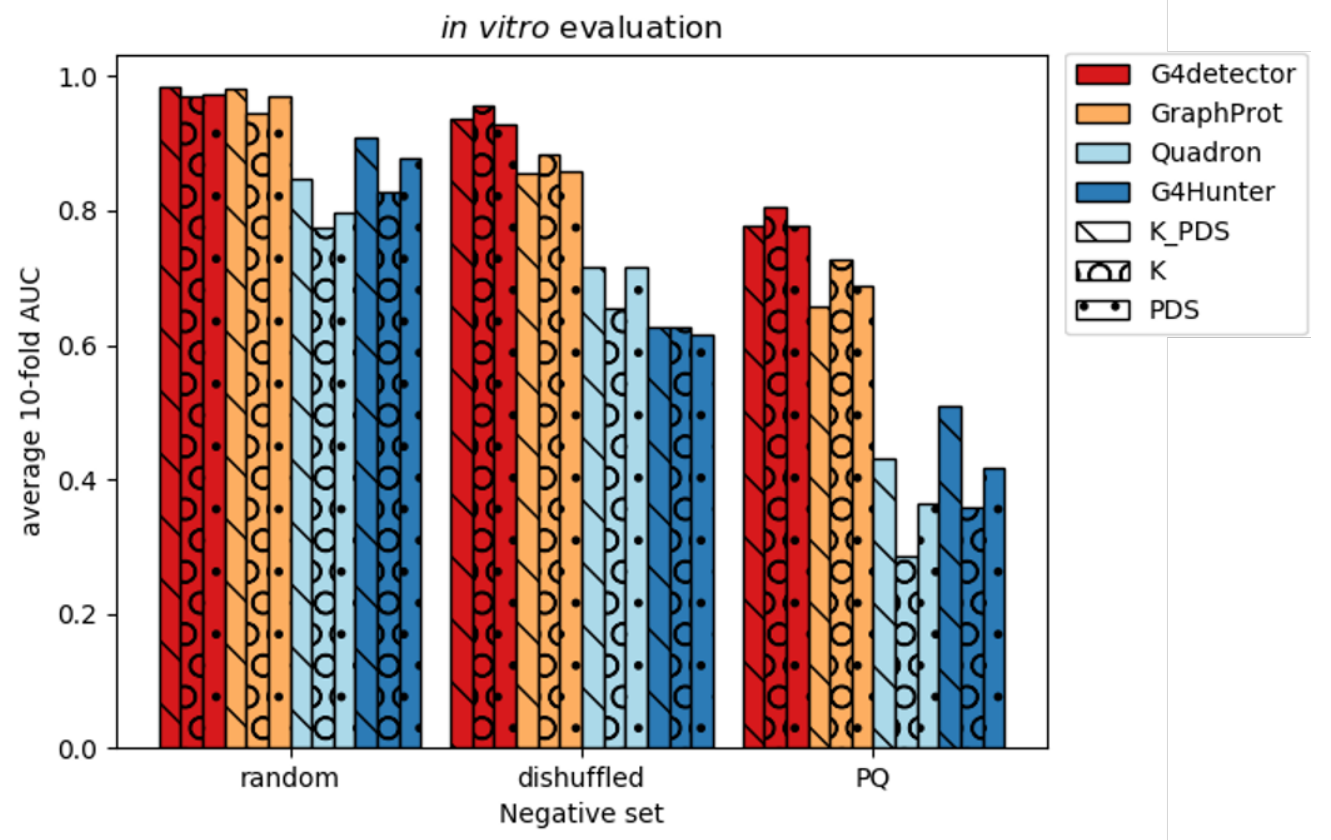

Figure 3: G4detector outperforms extant methods in predicting in vitro G4 formation.

We tested different methods in predicting in vitro G4 formation as measured by the G4-seq experiment. For G4detector and GraphProt, we performed 10-fold cross validation: we trained a model on nine folds of the in vitro data, and tested its accuracy on a held-out fold. This was repeated with three different negative sets: random genomic regions, dishuffled positives and predicted G-quadruplex, and for each of the experiments, which differed by the stabilizer (K, PDS, K_PDS). For Quadron and G4Hunter, we used the default trained model. For sequence set statistics, see Table 1.

\subsection{G4detector outperforms extant methods in predicting in vitro $\mathrm{G} 4$ formation}

To gauge the performance of G4detector, our deep neural network, compared to extant methods, we used the novel benchmark we created (see Subsection 2.1). For each of the G4 stabilizers (K, PDS and K_PDS), we performed 10 -fold cross validation. We split the data into ten equal folds, and for each fold we treated it as the test set while the rest were the training set. Performance was evaluated by area under the receiver operator curve (AUC), a common measure to gauge classification accuracy of balanced sets. We report the average AUC over the ten folds.

According to the results, as summarized in Figure 3, G4detector outperforms all competing methods in predicting in vitro G4 formation. GraphProt comes second in place after G4detector. We believe that it is due to the fact that it learned the G4 features from the same data as it is tested in the same 10-fold manner. Quadron, on the other hand, while trained on the same data, is based on predicting a mismatch score as opposed to a classification setting as in our case. This may explain why it is outperformed by both G4detector and GraphProt in all stabilizers and negative sets. Last, G4Hunter is also outperformed by G4detector and GraphProt on all the tasks. We assume that it is due to the fact that its computational model is too simplistic, as it uses only a regular expression to detect potential G4s.
As might be expected, when comparing the performance over different negative sets, the best performance was observed on the random negative set. It is the simplest of all three proposed classification problems, since it is highly unlikely for a sequence in this negative set to be guanine-rich (the human genome is AT-rich, Table 1), which is a key property of G4-forming sequences. When moving to shuffled sequences, they preserve the same nucleotide statistics, but lack the correct ordering to form a G4. We observed a decrease in accuracy for all methods, but still not as bad as the PQ negative set scenario. We observed the worst performance when training and testing on the PQ negative set. The PQ negative set is constructed out of areas in the human genome containing a G-rich regular expression (see Subsection 2.1). Due to the overlap with the test set, we expect some similarity between this negative set and the positive set, that most probably contains similar G-rich expressions.

\subsection{G4detector outperforms extant methods in predicting in vivo $\mathrm{G} 4$ formation}

To gauge the performance of G4detector, our neural network, compared to extant methods on predicting in vivo G4 formation, we used the G4 ChIP-seq dataset [14]. We trained nine binding models, each with a different stabilizer (K, PDS, K_PDS) and a different negative set (random, dishuffle, $\mathrm{PQ}$ ). Each binding model was trained on 


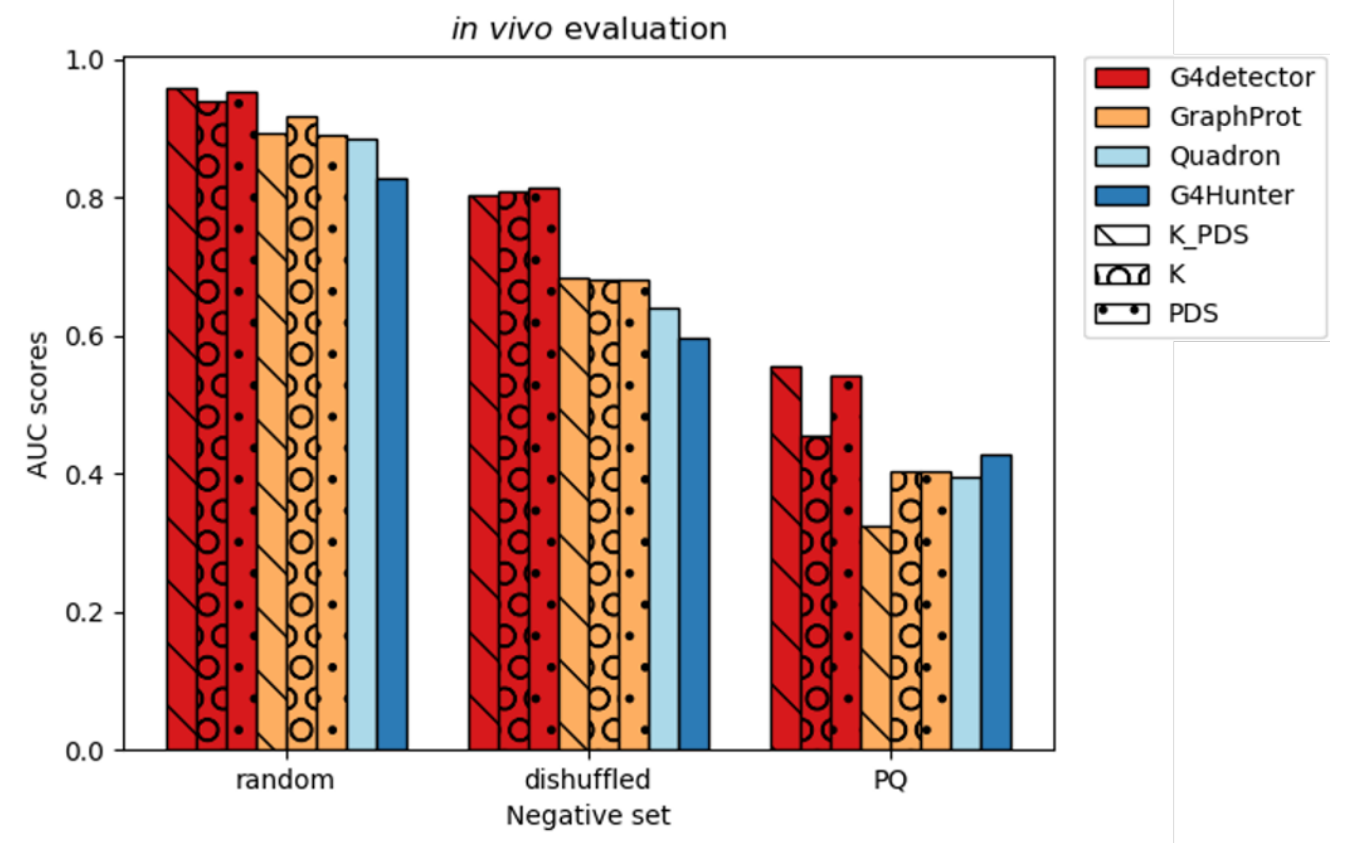

Figure 4: G4detector outperforms extant methods in predicting in vivo G4 formation.

We tested different methods in predicting in vivo G4 formation as measured by the G4 ChIP-seq experiment. In each test, the negative set is different: random genomic regions, dishuffled positives and predicted quadruplexes. For G4detector and GraphProt, we learned a model based on three high-throughput in vitro datasets (differed by stabilizer: K, PDS, K_PDS) using the same control (negative) set. For Quadron and G4Hunter, we used the default trained model. For sequence set statistics, see Table 1.

a complete in vitro dataset, and tested on the G4 ChIP-seq dataset augmented by the same type of negative set. To train the PQ models, during the training phase we held out 8,954 sequences from the PQ negative dataset, which is equal to the size of the positive dataset, and used that held-out set to test the model along with our positive set. We report the performance in predicting in vivo binding by AUC, an appropriate metric for balanced positive and negative sets, as in our case. For complete benchmarking details, see Subsection 2.1, and for sequence statistics see Table 1.

The results of predicting in vivo G4 formation show that G4detector performs the best on all three negative sets, and for each of the in vitro experiments it was trained on. As in the in vitro case, we observe an overall decrease in performance for all methods and models, moving from random to dishuffled to $P Q$, indicating that the $P Q$ negative set constitutes the greatest computational challenge. Note that on the PQ negative set, all three competing methods: GraphProt, Quadron and G4Hunter, achieve an AUC below 0.5, which corresponds to a random guess. Only our method, G4detector, was able to retrieve biologically meaningful features from the in vitro data that were relevant to the in vivo domain for the K_PDS and PDS stabilizers.

\subsection{G4detector identifies key sequence features to predict $\mathbf{G} 4$ formation}

We used the integrated gradients method to visualize the key features G4detector identifies on one given sequence. Figure 5 portrays the attribution scores evaluated using three different models, each differs by the negative set.

The attribution scores indicate that the models trained on the random and dishuffled negative sets are based on guanine-rich subsequences. The relevant guanines reside in continuous stretches. We also observed that for the model trained with the random negative set, higher attribution scores are given to pairs of guanine stretches seperated by one nucleotide. On the other hand, for the model trained on the PQ negative set, we see that similar genomic areas receive negative attributions. We suspect that the PQ-based model learned to identify the negative set, containing the regular expression, as opposed to identifying features that characterize G4s.

\subsection{G4detector identifies key interactions among G4 sequence features}

To visualize interactions between positions inside the G4 sequence, we used in silico mutagenesis. We calculated the difference between the sum of predicted effect of two SNPs compared to the predict effect of the corresponding DNP. Deviations from the additive model 


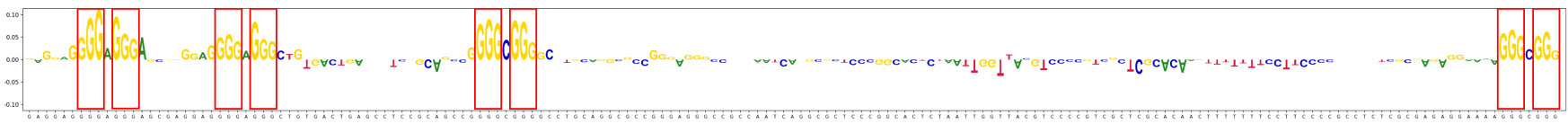

(a) random

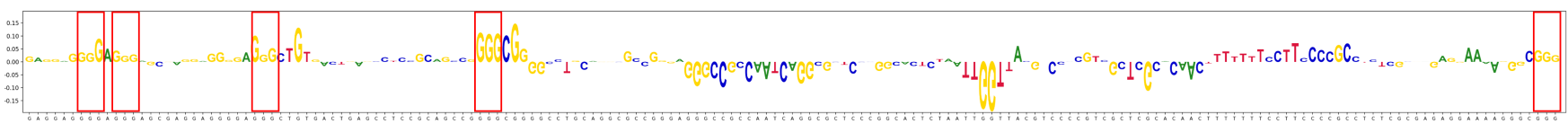

(b) dishuffled

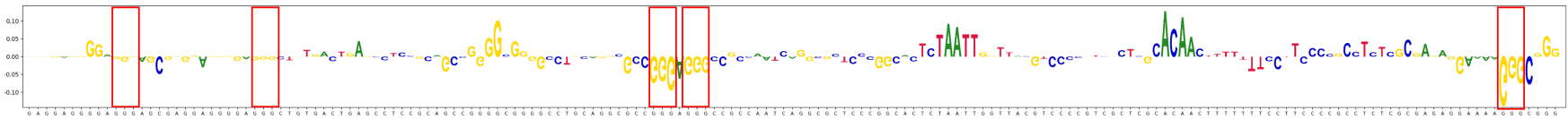

(c) $P Q$

Figure 5: G4detector identifies key features within the data.

Example of attribution scores assigned by the integrated gradients method for models trained on the positive G4-seq in vitro K_PDS set. (a) and (b) show that G-stretches received relatively high scores for models trained with the random and the dishuffled negative sets, respectively, whereas for the model trained with the PQ negative sets (c), G-stretches received negative scores.

$\begin{array}{lllllllllll}0 & 2 & 3 & 4 & 5 & 6 & 7 & 8 & 9 & 1011 & 121314151617181920212223242526272829\end{array}$

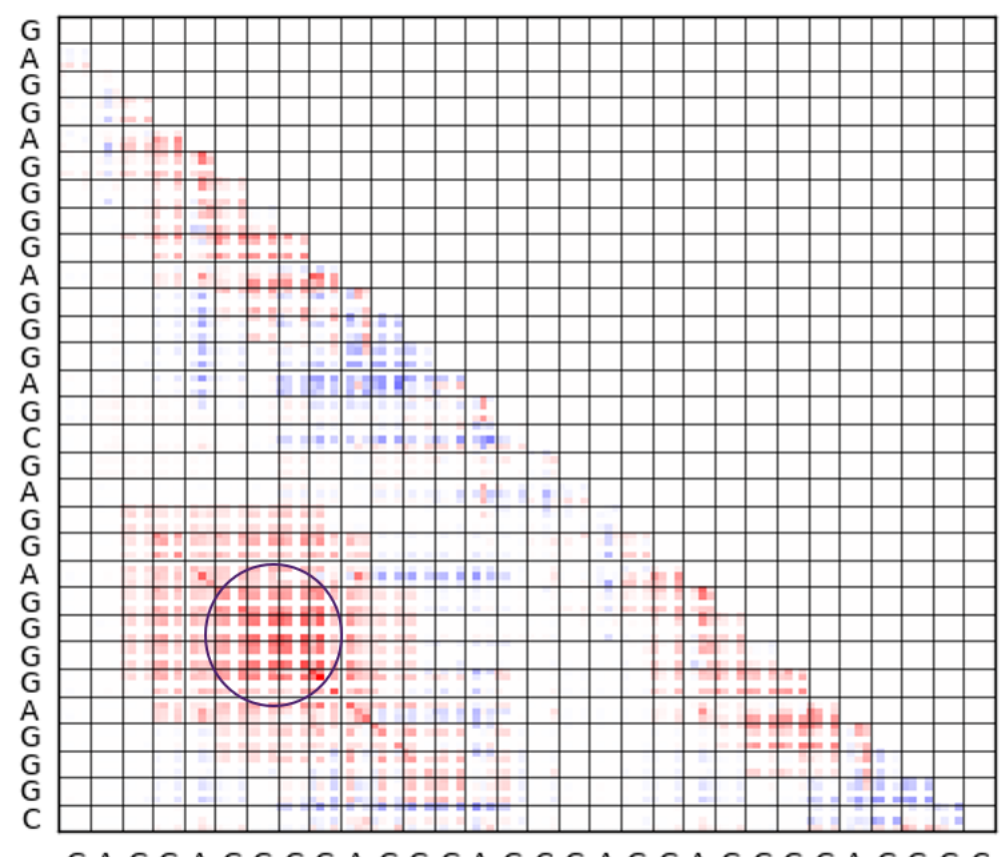

G A G G A G G G A G G A G C A G G A G G GA G G C
0.020

$-0.015$

$-0.010$

$-0.005$

$-0.000$

$-0.005$

$-0.010$

$-0.015$

$-0.020$

Figure 6: G4detector identifies key interactions among G4 sequence features.

An interaction map deduced from the model trained on the random negative set with K_PDS stabilizer. Each cell $(i, j)$ is the difference between the sum of predicted probabilities of SNP mutations in those positions compared to predicted value when both positions are mutated (i.e., DNP). In the circled region in the map, a strong interaction between G4 nucleotides was identified. 
are a way to identify interacting positions. We generated the mutation maps shown in Figure 6 using the first 30 nucleotides from the sequence used in Figure 5.

As can be seen in Figure 6, the model can successfully identify interacting G4 nucleotides. The map generated according to the random model highlights two likely interacting G4s. We observe extreme deviation from the additive model in the intersection between positions 5-8 and 21-24, where a subsequence of four guanines is present. We speculate that while mutation at one of these positions highly disturbs the G4 structure, adding a second mutation does not have the same disruptive effect as the sum of two single mutations. On contrast to the G4 subsequnce, in the linker regions we observe an additive effect, i.e., the difference between the sum of SNPs and the corresponding DNP is approximately zero.

\section{DISCUSSION}

In this paper, we present a novel method, G4detector, to predict G4-quadruplexes based on high-throughput data. To the best of our knowledge, G4detector is the first method to treat the problem as a classification problem, i.e., distinguishing G4 sequences vs. nonG4 sequences. To demonstrate the effectiveness of G4detector, we generated new benchmarks based on recently published datasets. These will be valuable for any future study for the development of new software and algorithms for this task.

We compared our algorithm to three extant methods and outperformed all of them. First, we compared to G4Hunter, which is based on simple rules of regular expression and nucleotide counting, to detect G4 formation potential. It is not based on learning from experimental data. Second, we compared to Quadron, which was developed to predict G4 formation 'intensity', measured as the number of mismatches identified in the G4-seq experimental protocol. While Quadron may solve the regression problem effectively, it was not on par with our method in solving the classification problem. Last, we compared to GraphProt in its sequence only mode and have demonstrated the power of deep neural networks compared to classic machine learning methods (e.g., support vectors) that use $k$-mer sequence features.

There are several caveats in our study. First, the type of negative set is critical in the definition of the problem. To circumvent our inability to generate a real biological control, we used three different negative sets. We demonstrated that our method, G4detector, outperforms on all three, both in vitro and in vivo. Second, while deep neural networks are very effective as prediction black boxes, they are difficult to interpret. To tackle this problem, we used a recently developed method, integrated gradients, to visualize the most important features in a given input sequence. Moreover, we successfully visualized interactions identified by the G4detector model by in silico mutagenesis analysis.

We see several paths to extend this work in the future. First, similarly to Quadron, we would like to explore the problem of predicting the strength of the G-quadruplex structure. This can be done based on the 'mismatch' level as measured in the G4-seq protocol. Second, we hope to improve our negative set to be based more on experimental data, rather than being a synthetic one. Last, we plan to apply G4detector on whole genomes and ask what is biologically common to regions in the mouse genome, for example, that form G4s.

To conclude, the new experimental data of G4 DNA formations generated recently under several studies posed new computational challenges that we tackle in this paper. These include analyzing the data, expressed as a list of DNA sequences annotated as Gquadruplexes and non-G4s, and inferring a predictive model from the data to enable predictions of G-quadruplex formation in new DNA sequences. We used the latest advancements in the machine learning world, deep learning, which is especially accustomed to picking up local features and interactions between them. Testing G4detector models on independent high-throughput datasets demonstrated its accuracy, effectiveness and ability to learn relevant biological signals from high-throughput sequencing data. We believe that our method, G4detector, will advance the research in the G-quadruplex field by providing researches with a new tool to predict G4s from DNA sequence alone.

\section{ACKNOWLEDGMENTS}

We gratefully acknowledge the support of Intel Corporation for giving access to the Intel ${ }^{\circledR}$ AI DevCloud platform used for part of this work.

\section{REFERENCES}

[1] Stephen F Altschul and Bruce W Erickson. 1985. Significance of nucleotide sequence alignments: a method for random sequence permutation that preserves dinucleotide and codon usage. Molecular biology and evolution 2, 6 (1985), 526538.

[2] Amina Bedrat, Laurent Lacroix, and Jean-Louis Mergny. 2016. Re-evaluation of G-quadruplex propensity with G4Hunter. Nucleic acids research 44, 4 (2016), 1746-1759.

[3] Giulia Biffi, David Tannahill, John McCafferty, and Shankar Balasubramanian. 2013. Quantitative visualization of DNA G-quadruplex structures in human cells. Nature chemistry 5, 3 (2013), 182

[4] Giulia Biffi, David Tannahill, Jodi Miller, William J Howat, and Shankar Balasubramanian. 2014. Elevated levels of G-quadruplex formation in human stomach and liver cancer tissues. PloS one 9, 7 (2014), e102711.

[5] Matthew L Bochman, Katrin Paeschke, and Virginia A Zakian. 2012. DNA secondary structures: stability and function of G-quadruplex structures. Nature Reviews Genetics 13, 11 (2012), 770

[6] Václav Brázda, Jan Kolomazník, Jiř́ Lỳsek, Martin Bartas, Miroslav Fojta, Jiří Št'astnỳ, and Jean-Louis Mergny. 2019. G4Hunter web application: a web server for G-quadruplex prediction. Bioinformatics (2019).

[7] David R Calabrese, Xiang Chen, Elena C Leon, Snehal M Gaikwad, Zaw Phyo, William M Hewitt, Stephanie Alden, Thomas A Hilimire, Fahu He, Aleksandra M Michalowski, et al. 2018. Chemical and structural studies provide a mechanistic basis for recognition of the MYC G-quadruplex. Nature communications 9, 1 (2018), 4229.

[8] David R Calabrese, Katherine Zlotkowski, Stephanie Alden, William M Hewitt, Colleen M Connelly, Robert M Wilson, Snehal Gaikwad, Lu Chen, Rajarshi Guha, Craig J Thomas, et al. 2018. Characterization of clinically used oral antiseptics as quadruplex-binding ligands. Nucleic acids research 46, 6 (2018), 2722-2732.

[9] Vicki S Chambers, Giovanni Marsico, Jonathan M Boutell, Marco Di Antonio, Geoffrey P Smith, and Shankar Balasubramanian. 2015. High-throughput sequencing of DNA G-quadruplex structures in the human genome. Nature biotechnology 33, 8 (2015), 877.

[10] José Almeida Cruz and Eric Westhof. 2009. The dynamic landscapes of RNA architecture. Cell 136, 4 (2009), 604-609.

[11] Jeffery T Davis. 2004. G-quartets 40 years later: from 5'-GMP to molecular biology and supramolecular chemistry. Angewandte Chemie International Edition 43, 6 (2004), 668-698.

[12] Johanna Eddy and Nancy Maizels. 2006. Gene function correlates with potential for G4 DNA formation in the human genome. Nucleic acids research 34, 14 (2006), 3887-3896.

[13] Verónica González and Laurence H Hurley. 2010. The C-terminus of nucleolin promotes the formation of the c-MYC G-quadruplex and inhibits c-MYC promoter activity. Biochemistry 49, 45 (2010), 9706-9714. 
[14] Robert Hänsel-Hertsch, Dario Beraldi, Stefanie V Lensing, Giovanni Marsico, Katherine Zyner, Aled Parry, Marco Di Antonio, Jeremy Pike, Hiroshi Kimura, Masashi Narita, et al. 2016. G-quadruplex structures mark human regulatory chromatin. Nature genetics 48, 10 (2016), 1267.

[15] Robert Hänsel-Hertsch, Marco Di Antonio, and Shankar Balasubramanian. 2017. DNA G-quadruplexes in the human genome: detection, functions and therapeutic potential. Nature Reviews Molecular Cell Biology 18, 5 (2017), 279.

[16] Alexander Henderson, Yuliang Wu, Yu Chuan Huang, Elizabeth A Chavez, Jesse Platt, F Brad Johnson, Robert M Brosh, Dipankar Sen, and Peter M Lansdorp. 2013. Detection of G-quadruplex DNA in mammalian cells. Nucleic acids research 42,2 (2013), 860-869.

[17] Naozuni Hiranuma. 2017. Integrated Gradients. (2017). https://github.com/ hiranumn/IntegratedGradients

[18] Julian L Huppert and Shankar Balasubramanian. 2005. Prevalence of quadruplexes in the human genome. Nucleic acids research 33, 9 (2005), 2908-2916.

[19] Oleg Kikin, Lawrence D’Antonio, and Paramjeet S Bagga. 2006. QGRS Mapper: a web-based server for predicting G-quadruplexes in nucleotide sequences. Nucleic acids research 34, suppl 2 (2006), W676-W682.

[20] Diederik P Kingma and Jimmy Ba. 2014. Adam: A method for stochastic optimization. arXiv preprint arXiv:1412.6980 (2014).

[21] Sebastian LB König, Amanda C Evans, and Julian L Huppert. 2010. Seven essential questions on G-quadruplexes. Biomolecular concepts 1, 2 (2010), 197-213.

[22] Laurent Lacroix. 2018. G4HunterApps. Bioinformatics (2018).

[23] Andrew N Lane, J Brad Chaires, Robert D Gray, and John O Trent. 2008. Stability and kinetics of G-quadruplex structures. Nucleic acids research 36, 17 (2008), 5482-5515.

[24] Daniel Maticzka, Sita J Lange, Fabrizio Costa, and Rolf Backofen. 2014. GraphProt: modeling binding preferences of RNA-binding proteins. Genome biology 15, 1 (2014), R17.

[25] Pierre Murat and Shankar Balasubramanian. 2014. Existence and consequences of G-quadruplex structures in DNA. Current opinion in genetics \& development 25 (2014), 22-29.

[26] Aaron R Quinlan. 2014. BEDTools: the Swiss-army tool for genome feature analysis. Current protocols in bioinformatics 47, 1 (2014), 11-12.
[27] Daniela Rhodes and Hans J Lipps. 2015. G-quadruplexes and their regulatory roles in biology. Nucleic acids research 43, 18 (2015), 8627-8637.

[28] Aleksandr B Sahakyan, Vicki S Chambers, Giovanni Marsico, Tobias Santner, Marco Di Antonio, and Shankar Balasubramanian. 2017. Machine learning model for sequence-driven DNA G-quadruplex formation. Scientific reports 7, 1 (2017), 14535 .

[29] Dipankar Sen and Walter Gilbert. 1988. Formation of parallel four-stranded complexes by guanine-rich motifs in DNA and its implications for meiosis. Nature 334, 6180 (1988), 364.

[30] Adam Siddiqui-Jain, Cory L Grand, David J Bearss, and Laurence H Hurley. 2002. Direct evidence for a G-quadruplex in a promoter region and its targeting with a small molecule to repress c-MYC transcription. Proceedings of the National Academy of Sciences 99, 18 (2002), 11593-11598.

[31] Mukund Sundararajan, Ankur Taly, and Qiqi Yan. 2017. Axiomatic attribution for deep networks. In Proceedings of the 34th International Conference on Machine Learning-Volume 70. JMLR. org, 3319-3328.

[32] Wesley I Sundquist and Aaron Klug. 1989. Telomeric DNA dimerizes by formation of guanine tetrads between hairpin loops. Nature 342, 6251 (1989), 825.

[33] Alan K Todd, Matthew Johnston, and Stephen Neidle. 2005. Highly prevalent putative quadruplex sequence motifs in human DNA. Nucleic acids research 33, 9 (2005), 2901-2907.

[34] Anna Varizhuk, Nikolay Ilyinsky, Igor Smirnov, and Galina Pozmogova. 2016. G4 aptamers: trends in structural design. Mini reviews in medicinal chemistry 16, 16 (2016), 1321-1329.

[35] M Neale Weitzmann, Kerry J Woodford, and Karen Usdin. 1996. The developmen and use of a DNA polymerase arrest assay for the evaluation of parameters affecting intrastrand tetraplex formation. Fournal of Biological Chemistry 271, 34 (1996), 20958-20964.

[36] James R Williamson. 1994. G-quartet structures in telomeric DNA. Annual review of biophysics and biomolecular structure 23, 1 (1994), 703-730.

[37] Qinhu Zhang, Lin Zhu, and De-Shuang Huang. 2018. High-Order Convolutional Neural Network Architecture for Predicting DNA-Protein Binding Sites. IEEE/ACM transactions on computational biology and bioinformatics (2018). 